\title{
LIMITS OF SOLUTIONS OF VOLTERRA INTEGRAL EQUATIONS ${ }^{1}$
}

BY R. C. MAC CAMY AND R. L. SMITH

Communicated by Richard Goldberg, January 14, 1975

Consider the Volterra integral equation

$$
u(t)=-\int_{0}^{t} A(t-\tau) g(u(\tau)) d \tau+f(t), \quad t>0,
$$

on a Hilbert space $H . A(t)$ is a family of bounded, linear, selfadjoint opera on $H$ and $g$ is a nonlinear bounded map from $H$ into itself. If $f(t) \rightarrow f_{0}(t)$ as $t \rightarrow \infty$ then

$$
u_{0}(t)=-\int_{0}^{\infty} A(\tau) g\left(u_{0}(t-\tau)\right) d \tau+f_{0}(t), \quad t>0,
$$

will be called a limit equation for (E). The following result appears in [7] .

Theorem (Miller). Let $H=R^{n}$. Suppose $A \in L_{1}(0, \infty), f: R^{+} \rightarrow$ $R^{n}$ is bounded and uniformly continuous, $g$ is continuous. Let (E) have a bounded solution $u$ on $R^{+}$. Then there exist a solution $u_{0}$ of $\left(\mathrm{E}_{0}\right)$ and a sequence $t_{n} \rightarrow \infty$ such that $u\left(t+t_{n}\right) \rightarrow u_{0}(t)$ as $n \rightarrow \infty$.

We give a result complementary to Miller's. We give conditions on $A$ and $g$ which guarantee that if $\left(\mathrm{E}_{0}\right)$ has a bounded solution then all solutions of (E) tend to $u_{0}$ as $t \rightarrow \infty$.

Our hypotheses are taken from [5]. We assume that $g$ is continuous, bounded with $g(0)=0$ and that

$$
\langle g(u)-g(v), u-v\rangle \geqslant m\|u-v\|^{2} \text { for some } m>0 \text {. }
$$

We assume that $A \in C^{(2)}[0, \infty), A^{(k)} \in L_{1}(0, \infty), k=0,1,2 . A$ also is to satisfy

$$
\begin{gathered}
\langle A(0) u, u\rangle \geqslant \alpha\|u\|^{2},\langle\dot{A}(0) u, u\rangle \leqslant-\beta\|u\|^{2}, \quad \alpha>0, \quad \beta>0, \\
\text { given any } N, \text { there exists } \delta(N)>0 \text { such that } \\
\left\langle\operatorname{Re} A^{\wedge}(i \eta) u, u\right\rangle \geqslant \delta(N)\|u\|^{2} \text { for all }|\eta| \leqslant N .
\end{gathered}
$$


In (3) $A^{\wedge}$ (s) is the Laplace transform of $A$. (See [6] for a comparison of (3) with the monotonicity and convexity conditions of [4] .) $f$ is to satisfy

$$
f(t)=f_{0}(t)+h(t) \quad \text { where } h \in L_{1}(0, \infty) \cap L_{2}(0, \infty) \text {. }
$$

THEOREM (1). Suppose $\left(\mathrm{E}_{0}\right)$ has a solution which is bounded on $R^{+}$. Then any solution of $(\mathrm{E})$ satisfies $u(t)-u_{0}(t) \rightarrow 0$ as $t \rightarrow \infty$.

The proof involves ideas from [5]. (E) can be solved for $g(u)$. $\left(\mathrm{E}_{0}\right)$ can be written in the form (E) with a forcing term depending on $u_{0}$ and the resulting equation solved for $g\left(u_{0}\right)$. One subtracts the resulting equations, multiplies by $u-u_{0}$ and integrates from 0 to $T$. Conditions (1)-(4) then yield an energy estimate which shows that $u-u_{0} \in L_{\infty}(0, \infty) \cap L_{2}(0, \infty)$. (E) and $\left(\mathrm{E}_{0}\right)$ can then be used to establish that $u-u_{0}$ is uniformly continuous, hence $u-u_{0} \rightarrow 0$ as $t \rightarrow \infty$.

There are two immediate applications.

THEOREM (2). Let the hypotheses of Theorem (1) hold and suppose $f_{0}(t)$ in (4) equals $f_{0}$ a constant. Then $\left(\mathrm{E}_{0}\right)$ has a constant solution $u_{0}$ and all solutions of $(\mathrm{E})$ tend to $u_{0}$ as $t \rightarrow \infty$.

Proof. We need only show that $\left(\mathrm{E}_{0}\right)$ has a constant solution. From (3) it follows that $A=A^{\wedge}(0)$ has a positive selfadjoint square root. The equation to be solved can be reduced to

$$
v_{0}+A^{1 / 2} g\left(A^{1 / 2} v_{0}\right)=v_{0}+F\left(v_{0}\right)=A^{-1 / 2} f_{0} .
$$

We have

$$
\begin{aligned}
\left\langle F\left(v_{0}\right)-F\left(v_{0}^{\prime}\right), v_{0}-v_{0}^{\prime}\right\rangle & =\left\langle g\left(A^{1 / 2} v_{0}\right)-g\left(A^{1 / 2} v_{0}^{\prime}\right), A^{1 / 2} v_{0}-A^{1 / 2} v_{0}^{\prime}\right\rangle \\
& \geqslant m\left\|A^{1 / 2}\left(v_{0}-v_{0}^{\prime}\right)\right\|^{2}>0 .
\end{aligned}
$$

Hence $F$ is a continuous monotone operator and it follows by a result of Minty [8] that (5) has a solution.

The second application concerns the existence of periodic limits. Let $P_{T}$ denote the set of all $T$-periodic functions $u$ on $L_{2}((-\pi, \pi): H)$. For $u \in P_{T}$ we have a Fourier series $u=\Sigma_{-\infty}^{+\infty} u_{n} e^{\text {int }}$. Consider the linear operator $A$ defined by $A v(t)=\int_{0}^{\infty} A(\tau) v(t-\tau) d \tau$. For $v \in P_{T}$,

$$
A v=\Sigma A^{\wedge}(\text { in }) v_{n} e^{\text {int }}
$$

it is slown in [5] that the hypotheses on $A$ imply that $\left\|A^{\wedge}(s)\right\|=O\left(s^{-1}\right)$ 
as $s \rightarrow \infty$. From this one concludes that $A$ is a bounded linear map from $P_{T}$ into $P_{T} \cap L_{\infty}((-\pi, \pi): H)$. If $H$ is finite dimensional the map from $P_{T}$ into itself is compact. The same remarks hold for the nonlinear map $v \rightarrow$ $\int_{0}^{\infty} A(\tau) g(v(t-\tau)) d \tau$ if $g$ satisfies

$$
\|g(v)\| \leqslant K\|v\| .
$$

Assume now that $f_{0} \in P_{T} \cap L_{\infty}((-\pi, \pi): H)$ and the hypotheses of Theorem (1) hold.

THEOREM (3). Suppose $g(u)=L u$ where $L$ is a bounded linear selfadjoint operator. Then $\left(\mathrm{E}_{0}\right)$ has a solution $u_{0} \in P_{T} \cap L_{\infty}((-\pi, \pi): H)$ and all solutions of $(\mathrm{E})$ tend to $\left(\mathrm{E}_{0}\right)$ as $t \rightarrow \infty$.

PRoof. A solution can be found in the form $u_{0}=\Sigma u_{n}^{0} e^{i n t}$ where $\left(I+A^{\wedge}(i n) L\right) u_{n}^{0}=f_{n}^{0}, f(t)=\Sigma f_{n}^{0} e^{\text {in } t}$. The hypotheses on $A$ and $L$ guarantee that for each $n,\left(I+A^{\wedge}(i n) L\right)^{-1}$ exists as a bounded operator with $\left\|\left(I+A^{\wedge}(i n)\right)^{-1}\right\| \leqslant J$ (see [5]). It follows easily that the series yields the desired solution. If $f_{0}$ is continuous then $u_{0}$ is continuous.

Theorem (3) can be used with a perturbation argument and the contractive mapping principle to yield the following result.

Theorem (4). Let $g(u)=L u+\epsilon h(u)$, where $L$ is as in Theorem (3), $h(0)=0, h$ satisfies (1) and $h$ is globally Lipschitz. Then for $\epsilon$ sufficiently small $\left(\mathrm{E}_{0}\right)$ has a solution $u_{0}$ and all solutions of $(\mathrm{E})$ tend to $u_{0}$ as $t \rightarrow \infty$.

On finite dimensional spaces one can use the Schauder theorem to establish the existence of solutions of $\left(\mathrm{E}_{0}\right)$ if $g(u)=o(\|u\|)$ as $\|u\| \rightarrow \infty$ (see [1] for a related result) or if $g(u)=\epsilon h(u)$ where $\|h(u)\| \leqslant k(\|u\|+1$ ) and $\epsilon$ is small. If one has a priori bounds for solutions of (E) then one can eliminate the growth condition on $g$ by making use of Miller's result and Theorem 1. One situation in which this idea can be applied is that of the following lemma which is given for $H=R^{1}$ in [3] .

Lemma. Suppose that $f$ in $(\mathrm{E})$ is in $L_{\infty}((-\infty, \infty): H)$. Suppose further that the conditions of Theorem (1) hold and that there exists an $\alpha>0$ such that $e^{\alpha t} A(t)$ still satisfies the hypotheses of Theorem (1). Then there exists an $M>0$ such that all solutions of (E) satisfy $\|u(t)\| \leqslant M$ for all $t \geqslant 0$.

Similar results to those in this paper can be obtained for the differentiated version of $(E)$. These are related to the results of [2] . 


\title{
REFERENCES
}

1. V. E. Beneš and I. W. Sandberg, Applications of a theorem of Dubrovskil to the periodic responses on nonlinear systems, Bell System Tech. J. 43 (1964), 2855-2872. MR 32 \#8096.

2. S. I. Grossman and R. K. Miller, Nonlinear Volterra integro-differential systems with $L^{1}$ kernels, J. Differential Equations 13 (1973), 551-566.

3. M. J. Leitman and V. J. Mizel, Hereaitary laws and nonlinear integral equations on the line, J. Differential Equations (to appear).

4. J. L. Levin and J. A. Nohel, Perturbations of a nonlinear Volterra equation, Michigan Math. J. 12 (1965), 431-447. MR 32 \#336.

5. R. C. MacCamy, Remarks on frequency domain methods for Volterra integral equations, J. Math. Anal. Appl. (to appear)

6. R. C. MacCamy and J. S. W. Wong, Stability theorems for some functional equations, Trans. Amer. Math. Soc. 164 (1972), 1-37. MR 45 \#2432.

7. R. K. Miller, Nonlinear Volterra integral equations, Benjamin, New York, 1971.

8. G. J. Minty, Monotone (nonlinear) operators in Hilbert space, Duke Math. J. 29 (1962), 341-346. MR 29 \#6319.

DEPARTMENT OF MATHEMATICS, CARNEGIE-MELLON UNIVERSITY, PITTSBURGH, PENNSYLVANIA 15213

\section{BULLETIN OF THE}

AMERICAN MATHEMATICAL SOCIETY

Volume 81, Number 4, July 1975

\section{MAXIMA IN BROWNIAN EXCURSIONS}

\author{
BY KAI LAI CHUNG ${ }^{1}$
}

Communicated by Daniel W. Stroock, February 25, 1975

Let $\{X(t), t \geqslant 0\}$ be the standard one-dimensional Brownian motion starting at 0 . For $t>0$ define

$$
\begin{aligned}
T(t) & =\sup \{s \leqslant t \mid X(s)=0\} ; & T^{\prime}(t) & =\inf \{s \geqslant t \mid X(s)=0\} ; \\
L^{-}(t) & =t-T(t) ; & L(t) & =T^{\prime}(t)-T(t) ; \\
M^{-}(t) & =\max _{T(t) \leqslant s \leqslant t}|X(s)| ; & M(t) & =\max _{T(t) \leqslant s \leqslant T^{\prime}(t)}|X(s)| .
\end{aligned}
$$

The random time interval $\left(T(t), T^{\prime}(t)\right)$ is the excursion interval straddling $t$,

AMS (MOS) subject classifications (1970). Primary 60J65, 60J10, 60E05. function.

Key words and phrases. Brownian motion, excursion, last exit, entrance law, theta

${ }^{1}$ This work was supported in part by NSF grant GP 41710 at Stanford University. Copyright $\odot$ 1975, American Mathematical Society 